\title{
Effect of Extraction Time on Physiologically Important Constituents of Green Tea (Camellia sinensis) Using GC/MS
}

\author{
Mudasir Ahmad1, Adil Gani ${ }^{1 *}$, Waqas N Baba ${ }^{1}$, Asir Gani ${ }^{2}$, Wani SM1, Masoodi FA ${ }^{1}$, Asima Shah ${ }^{1}$ and Sajad A Rather \\ ${ }^{1}$ Department of Food Science and Technology, University of Kashmir, India \\ ${ }^{2}$ Department of Food Processing and Engineering, SLITE, Punjab, India
}

\begin{abstract}
Two types of green tea samples Fine and Superfine were subjected to varied extraction times (20, 40, and 120 minutes) at constant temperature of $90^{\circ} \mathrm{C}$. These tea extracts of were then subjected to GC-MS analysis. The phytochemical composition of these extracts showed some variations depending upon the type of sample possibly due to genetic, environmental and processing conditions. Among different time temperature combinations it was seen that a time period of 20-40 minutes was generally suitable for most of the samples for retaining a higher percentage of physiologically significant phytochemicals in the extract. Also pthalic acid, a toxic substance, was reported to be present in superfine variety, but aqueous extracts of fine tea variety did not yield any of it.
\end{abstract}

Keywords: Camellia sinensis; Extraction; Phytochemicals; GC-MS analysis; Caffeine; Pthalic acid

\section{Introduction}

Tea (Camellia sinensis) is native to the southern regions of China and parts of India, Laos, Thailand, Vietnam, and Myanmar [1]. Tea is said to have first been discovered as a drink and medicine in China around $2737 \mathrm{BC}$. It was then introduced to Japan during the early 13th century and to Europe in the 16th century, then to America, Africa and other regions of the world $[1,2]$. Tea is presently cultivated in over 30 countries around the world and the tea beverage is second only to water in terms of worldwide consumption [3]. It grows best in tropical and subtropical areas with adequate rainfall, good drainage, and slightly acidic soil. The worldwide popularity of tea has increased due to its potential health benefits against cardiovascular diseases and cancer as well as pharmaceutical potential such as anti-hypertensive, antiateriosclerotic, hypocholesteroladmic, and hypolipidemic properties mostly from activities of flavonoids present in tea [4-6]. The Green tea is also known for its unique aroma and characteristic flavor [7]. For a better understanding of the physiological and pharmacological effects of tea, it is essential to scrutinize its chemical composition. There exist volatile and non-volatile components in tea. A great deal of work about tea volatiles have been reported [8-10]. But as far as we know, the effect of time-of-extraction on volatile constituents of green tea have not yet been reported. In general, the analysis of volatile components is usually conducted by using gas chromatography (GC) and gas chromatography-mass spectrometry (GC/MS). Here we report the identification of the volatile constituents of two types of green tea using GC/MS technique and extraction behavior of physiologically important volatile components with respect to different time of extraction.

\section{Materials and Methods}

\section{Sample collection and preparation of extract}

The dried leaves of green tea samples viz., fine and superfine were purchased from the local market and stored at room temperature until use. The tea extracts of dried leaves were prepared using stirring water bath, $2 \mathrm{~g}$ of fresh dried leaves were extracted with $50 \mathrm{ml}$ pure water at constant temperature of $90^{\circ} \mathrm{C}$, for different times (20, 40 and $120 \mathrm{~min}$ ). The extracts obtained were concentrated under reduced pressure in a rotary evaporator (Rotary equitron).

\section{Instrument and chromatographic conditions}

The concentrated pure extract was vacuum dried in a vacuum oven at $60^{\circ} \mathrm{C}$ to get a powdered residue. The residue was dissolved in $20 \mathrm{ml}$ methanol and collected in corked glass test tubes. The extracts were analysed on a Shimadzu QP2010 Plus GC-MS system with 2010 GC. An Omega SPTm column (0.25 mm ID, film thickness $0.25 \mu \mathrm{m})$ was used with nitrogen as carrier gas. The injector temperature was $270^{\circ} \mathrm{C}$ with split ratio of 10.0. The GC oven temperature was programmed to hold at $100^{\circ} \mathrm{C}$ for 2 minutes and then increased to $200^{\circ} \mathrm{C}$ at $15^{\circ} \mathrm{C} /$ min and hold for 2 minutes and finally increased to $240^{\circ} \mathrm{C}$ at $20^{\circ} \mathrm{C} /$ min and hold for 18 minutes. Ion source temperature was $230^{\circ} \mathrm{C}$ and the interface temperature was set at $280^{\circ} \mathrm{C}$. Mass spectra were collected over the range of $\mathrm{m} / \mathrm{z}$ 40-650. Each compound was identified using WILEY library $(8 \mathrm{~L})$.

\section{Results and Discussions}

\section{Identification and analysis various of components}

GC-MS chromatograms of the aqueous extract of two types of green tea for different time of extraction are given Figures 1 and 2 . The number of phytochemical constituents as depicted by the peaks varied according to the type of tea sample (possibly due to varied environmental, genetic and processing conditions) and the time of extraction. On comparison of the mass spectra of the constituents with the library (WILEY8.LIB) the different compounds were characterized and identified (Tables 1 and 2). The relative percentages of some major compounds which are present in almost in all tea types are presented in Table 3. They were identified as, Methyltetradecanoate/ Myristic acid, Hexadecanoic acid/palmitic acid, Octadecanoic acid/

*Corresponding author: Adil Gani, Department of Food Science and Technology, University of Kashmir, India, Tel: 8803023830; E-mail: adil.gani@gmail.co

Received July 22, 2013; Accepted October 28, 2013; Published November 05, 2013

Citation: Ahmad M, Gani A, Baba WN, Gani A, Wani SM, et al. (2013) Effect of Extraction Time on Physiologically Important Constituents of Green Tea (Camellia sinensis) Using GC/MS. J Food Process Technol 4: 276. doi:10.4172/2157 7110.1000276

Copyright: $\odot 2013 \mathrm{Ahmad}$ M, et al. This is an open-access article distributed under the terms of the Creative Commons Attribution License, which permits unrestricted use, distribution, and reproduction in any medium, provided the original author and source are credited. 


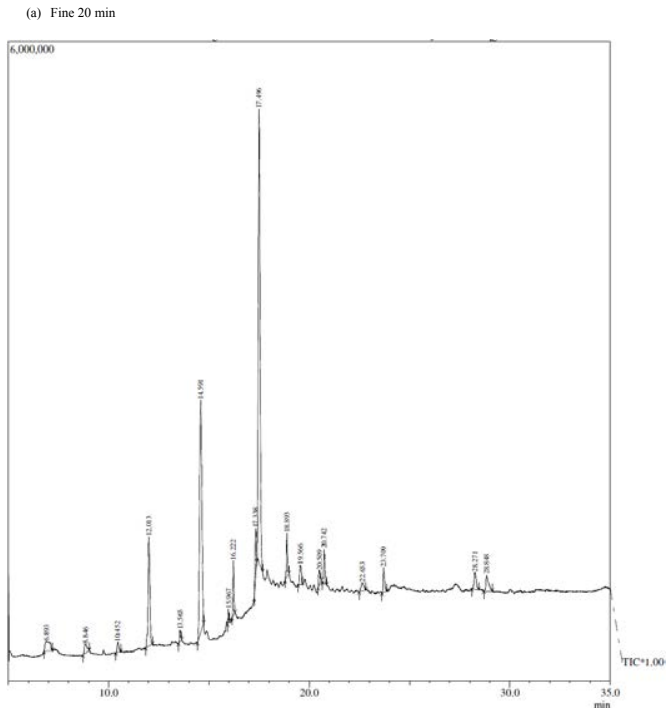

(b) Fine 40 min

$6,000,000$

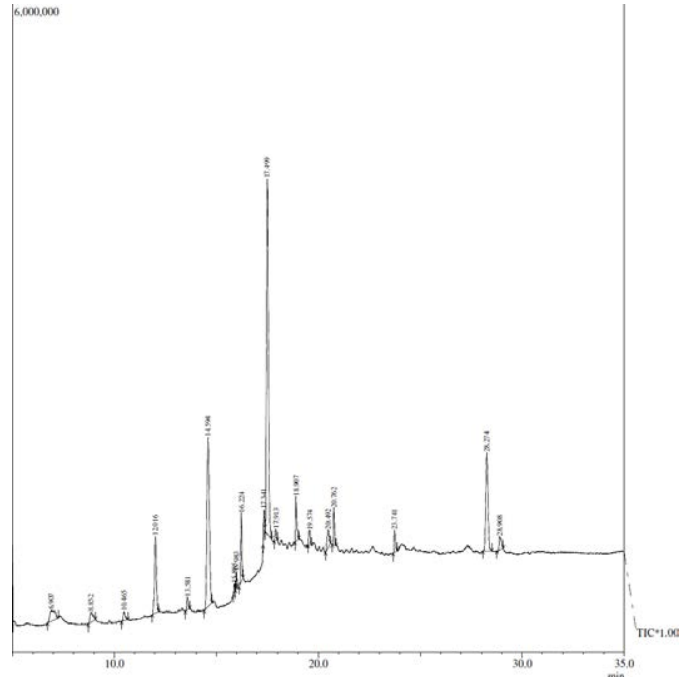

(c) Finel20 min.

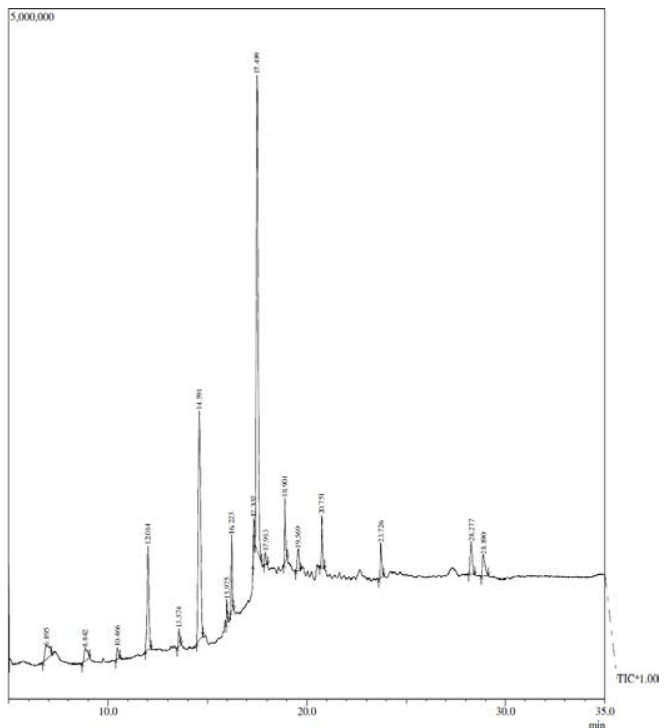

Figure 1: (a-c) Showing GCMS chromatographs of Fine green tea.

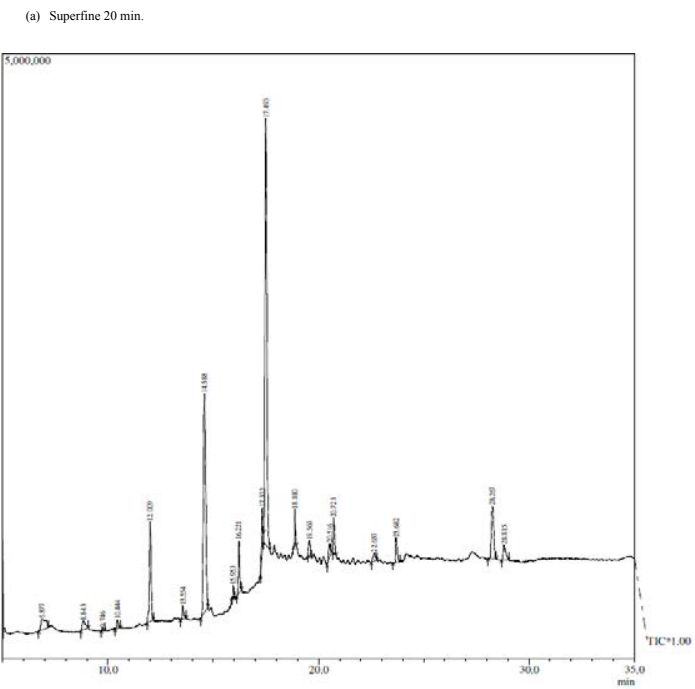

(b) Superfine 40 min

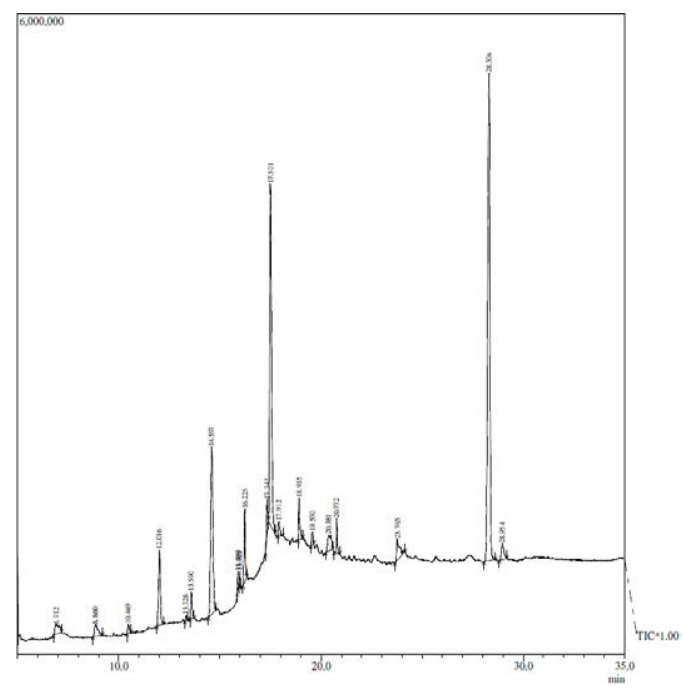

(c) Superfine 120 min.

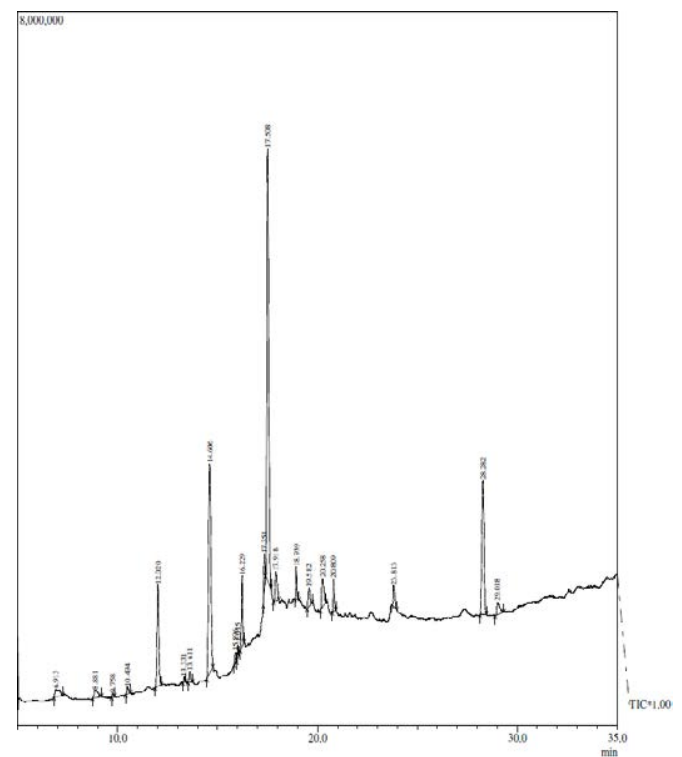

Figure 2: (a-c) Showing the GCMS chromatograph of superfine green tea. 
Citation: Ahmad M, Gani A, Baba WN, Gani A, Wani SM, et al. (2013) Effect of Extraction Time on Physiologically Important Constituents of Green Tea (Camellia sinensis) Using GC/MS. J Food Process Technol 4: 276. doi:10.4172/2157-7110.1000276

(a): Fine $20 \mathrm{~min}$

\begin{tabular}{|c|c|c|c|}
\hline Peak & R. time & Area $\%$ & Compound name \\
\hline 1 & 6.893 & 2.39 & Cyclooctasiloxane \\
\hline 2 & 8.846 & 1.21 & Cyclononasiloxane \\
\hline 3 & 10.452 & 0.82 & Cosamethylcyclodecasiloxane \\
\hline 4 & 12.013 & 8.74 & Methyl tetradecanoate/Myristic acid \\
\hline 5 & 13.565 & 0.67 & Cyclononasiloxane \\
\hline 6 & 14.591 & 24.77 & Hexadecanoic acid/palmitic acid \\
\hline 7 & 15.967 & 0.53 & 1H-Purin-6-amine \\
\hline 8 & 16.222 & 2.95 & 1,2-Benzenedicarboxylic acid \\
\hline 9 & 17.338 & 3.28 & Octadecanoic acid/Stearic acid \\
\hline 10 & 17.496 & 40.41 & 9-Octadecenoic acid/oleic acid \\
\hline 11 & 18.893 & 2.23 & 1H-Purin-6-amine \\
\hline 12 & 19.566 & 1.87 & 9,11-Octadecadienoic acid \\
\hline 13 & 20.509 & 1.13 & Acetic acid \\
\hline 14 & 20.742 & 1.96 & 1H-Purin-6-amine \\
\hline 15 & 22.653 & 1.21 & Docosanoic acid \\
\hline 16 & 23.709 & 1.81 & 1H-purin-6-amine \\
\hline 17 & 28.271 & 1.82 & 1H-Purine-2,6-dione/Caffein \\
\hline 18 & 28.848 & 2.2 & Cyclodecasiloxane \\
\hline
\end{tabular}

(b): Fine $40 \mathrm{~min}$

\begin{tabular}{|c|c|c|c|}
\hline Peak & RT F2 & F2A & Compound Name \\
\hline 1 & 6.907 & 2.88 & Cyclooctasiloxane \\
\hline 2 & 8.852 & 1.69 & Cyclononasiloxane \\
\hline 3 & 10.465 & 0.98 & Cyclodecasiloxane \\
\hline 4 & 12.016 & 6.85 & Methyl tetradecanoate/Myristic acid \\
\hline 5 & 13.581 & 0.99 & Cyclononasiloxane \\
\hline 6 & 14.594 & 20.6 & Hexadecanoic acid/palmitic acid \\
\hline 7 & 15.885 & 0.39 & Phenol, 2-methoxy-4-propenyl \\
\hline 8 & 15.983 & 0.76 & 1H-Purin-6-amine \\
\hline 9 & 16.224 & 4.24 & 1,2-Benzenedicarboxylic acid \\
\hline 10 & 17.341 & 1.57 & Octadecanoic acid/Stearic acid \\
\hline 11 & 17.499 & 35.13 & 9-Octadecenoic acid/oleic acid \\
\hline 12 & 17.913 & 0.91 & 9,12-Octadecadienoic Acid/Linoleic acid \\
\hline 13 & 18.907 & 2.57 & 1H-Purin-6-amine \\
\hline 14 & 19.574 & 1.46 & Cyclopropanebutanoic acid \\
\hline 15 & 20.492 & 2.2 & 9-Octadecenoic acid \\
\hline 16 & 20.762 & 2.19 & 1H-Purin-6-amine \\
\hline 17 & 23.741 & 1.64 & 1H-Purin-6-amine \\
\hline 18 & 28.274 & 11.47 & 1H-Purine-2,6-dione/Caffein \\
\hline 19 & 28.908 & 1.48 & Cyclononasiloxane \\
\hline
\end{tabular}

(c): Fine $120 \mathrm{~min}$.

\begin{tabular}{|l|l|l|l|}
\hline Peak & RTF6 & F6A & Compound Name \\
\hline 1 & 6.895 & 2.91 & Cyclooctasiloxane \\
\hline 2 & 8.842 & 1.94 & Bis(heptamethylcyclotetrasiloxy) hexamethyltrisiloxane \\
\hline 3 & 10.466 & 0.97 & Cyclodecasiloxane \\
\hline 4 & 12.014 & 7.87 & Methyl tetradecanoate/Myristic acid \\
\hline 5 & 13.574 & 1.04 & Cyclononasiloxane \\
\hline 6 & 14.591 & 23.21 & Hexadecanoic acid/palmitic acid \\
\hline 7 & 15.975 & 0.88 & 1 H-Purin-6-amine \\
\hline 8 & 16.223 & 3.82 & $1,2-$-Benzenedicarboxylic acid, Diethyl ester \\
\hline 9 & 17.337 & 1.56 & Octadecanoic acid/Stearic acid \\
\hline 10 & 17.499 & 39.33 & $9-$ Octadecenoic acid/oleic acid \\
\hline 11 & 17.913 & 0.85 & $9,12-$ Octadecadienoic acid, Linoleic acid \\
\hline 12 & 18.901 & 2.95 & 1 H-Purin-6-amine \\
\hline 13 & 19.569 & 1.97 & $\begin{array}{l}\text { 9,11-Octadecadienoic acid,Methyl trans-9,trans-11- } \\
\text { octadecadienoate }\end{array}$ \\
\hline 14 & 20.751 & 2.72 & 1H-Purin-6-amine \\
\hline 15 & 23.726 & 2.43 & 1H-Purin-6-amine \\
\hline 16 & 28.277 & 3.03 & 1H-Purine-2,6-dione/Caffein \\
\hline 17 & 28.89 & 2.54 & Cyclononasiloxane \\
\hline
\end{tabular}

Table 1(a-c) : Showing the identification and relative percentage of volatile compounds in Fine green tea at $90^{\circ} \mathrm{C}$ in 20,40 and 120 minutes time of extraction. (a): Super fine $20 \mathrm{~min}$

\begin{tabular}{|c|c|c|c|}
\hline Peak & R. time & Area\% & Name of compound \\
\hline 1 & 6.912 & 1.51 & Cyclooctasiloxane \\
\hline 2 & 8.86 & 1.44 & Cyclohexasiloxane \\
\hline 3 & 10.469 & 0.54 & Cyclononasiloxane \\
\hline 4 & 12.016 & 4.5 & Pentadecanoic acid \\
\hline 5 & 13.328 & 0.23 & Phenol \\
\hline 6 & 13.59 & 1.2 & Chlorocyclopentane \\
\hline 7 & 14.597 & 13.83 & Hexadecanoic acid, Palmitic acid, methyl ester \\
\hline 8 & 15.893 & 0.62 & Phenol \\
\hline 9 & 15.988 & 0.49 & $\begin{array}{l}\text { Cyclononasiloxane, octadecamethyl, } \\
\text { Octadeamethyl-cyclononasiloxane, } \\
\text { Octadecamethylcyclon }\end{array}$ \\
\hline 10 & 16.225 & 3.09 & Phthalic acid, diethyl ester \\
\hline 11 & 17.342 & 1.7 & Octadecanoic acid, methyl ester \\
\hline 12 & 17.501 & 22.99 & 9-Octadecenoic acid, Methyl ester, Oleic acid \\
\hline 13 & 17.912 & 0.85 & Linoleic acid, \\
\hline 14 & 18.915 & 1.58 & Cyclononasiloxane \\
\hline 15 & 19.57 & 0.77 & $\begin{array}{c}9,12-\text { Octadecadienoic acid }(Z, Z)-\text {, methyl ester } \$ \$ \\
\text { Linoleic acid, methyl ester }\end{array}$ \\
\hline 16 & 20.381 & 2.09 & Tetradecanoic acid $\$ \$$ Myristic acid \\
\hline 17 & 20.772 & 1.37 & $\begin{array}{l}\text { Cyclodecasiloxane, eicosamethyl, } \\
\text { Icosamethylcyclodecasiloxane }\end{array}$ \\
\hline 18 & 23.765 & 2.1 & Cyclooctasiloxane, Hexadecamethyl \\
\hline 19 & 28.306 & 37.7 & Caffein \\
\hline 20 & 28.954 & 1.41 & Cyclononasiloxane, octadecamethyl \\
\hline
\end{tabular}

(b): Superfine $40 \mathrm{~min}$

\begin{tabular}{|c|c|c|c|}
\hline Peak & R. time & Area\% & Name of compound \\
\hline 1 & 6.877 & 2.73 & Cyclooctasiloxane, Hexadecamethyl- \\
\hline 2 & 8.843 & 1.44 & Cyclohexasiloxane, dodecamethyl \\
\hline 3 & 9.746 & 0.19 & Tridecanoic acid, methyl ester \\
\hline 4 & 10.444 & 0.72 & Icosamethylcyclodecasiloxane \\
\hline 5 & 12.009 & 7.92 & Methyl tetradecanoate, Myristic acid, methyl ester \\
\hline 6 & 13.554 & 1.01 & Cyclononasiloxane, Octadecamethyl \\
\hline 7 & 14.588 & 23.87 & Hexadecanoic acid, Methyl Ester \\
\hline 8 & 15.953 & 0.54 & 1H-Purin-6-amine, [(2-Fluorophenyl)methyl] \\
\hline 9 & 16.221 & 3.28 & $\begin{array}{l}\text { 1,2-Benzenedicarboxylic acid, Diethyl ester, Phthalic } \\
\text { acid }\end{array}$ \\
\hline 10 & 17.332 & 2.87 & $\begin{array}{l}\text { Octadecanoic acid; Methyl Ester; Stearic acid Methyl } \\
\text { Ester }\end{array}$ \\
\hline 11 & 17.493 & 38.46 & 9-Octadecenoic acid, Oleic acid, methyl ester \\
\hline 12 & 18.88 & 1.52 & 1H-Purin-6-amine, [(2-Fluorophenyl)methyl] \\
\hline 13 & 19.569 & 1.5 & Cyclopropanebutanoic acid \\
\hline 14 & 20.516 & 1.19 & $\begin{array}{l}\text { Hexadecanoic acid, (2-Phenyl-1,3-dioxolan-4-yl) } \\
\text { methyl palmitate }\end{array}$ \\
\hline 15 & 20.723 & 2.05 & 1H-Purin-6-amine, [(2-Fluorophenyl)methyl] \\
\hline 16 & 22.657 & 1.03 & Methyl behenate, n-Docosanoic acid methyl ester \\
\hline 17 & 23.682 & 1.98 & 1H-Purin-6-amine, [(2-Fluorophenyl)methyl]- \\
\hline 18 & 28.267 & 5.79 & $\begin{array}{c}\text { 1H-Purine-2,6-dione, 3,7-dihydro-1,3,7-trimethyl-, Ca- } \\
\text { feina, Caffein, Caffine, Cafipel, Coffeine, Guaranine, } \\
\text { Koffein }\end{array}$ \\
\hline 19 & 28.815 & 1.9 & $\begin{array}{l}\text { 1H-Purin-6-amine, [(2-Fluorophenyl)methyl], Octadea- } \\
\text { methyl-cyclononasiloxane }\end{array}$ \\
\hline
\end{tabular}

(c): Superfine $120 \mathrm{~min}$

\begin{tabular}{|c|c|c|c|}
\hline Peak & R. time & Area\% & Name of compound \\
\hline 1 & 6.913 & 1.48 & Cyclooctasiloxane, hexadecamethyl- \\
\hline 2 & 8.881 & 1.26 & Cyclononasiloxane, octadecamethyl- \\
\hline 3 & 9.758 & 0.21 & Dodecanoic acid, methyl ester \\
\hline 4 & 10.494 & 0.57 & Icosamethylcyclodecasiloxane \\
\hline 5 & 12.02 & 7.25 & Tetradecanoic acid, Methyl ester \\
\hline 6 & 13.331 & 0.3 & Phenol, 2-Methoxy-4-(2-Propenyl) \\
\hline 7 & 13.611 & 0.68 & Cyclononasiloxane, Octadecamethyl \\
\hline
\end{tabular}




\begin{tabular}{|c|c|c|c|}
\hline 8 & 14.606 & 21.07 & Palmitic Acid, Hexadecanoic acid, Methyl ester \\
\hline 9 & 15.896 & 0.46 & Phenol, 2-methoxy-4-(1-propenyl \\
\hline 10 & 16.015 & 0.49 & Cyclononasiloxane, Octadecamethyl. \\
\hline 11 & 16.229 & 3.63 & Phthalic acid, diethyl ester \\
\hline 12 & 17.351 & 1.95 & Octadecanoic acid, Stearic acid \\
\hline 13 & 17.508 & 34.92 & $\begin{array}{c}\text { Oleic acid, methyl ester, 9-Octadecenoic acid } \\
\text { methyl ester }\end{array}$ \\
\hline 14 & 17.918 & 2.6 & Linoleic acid, methyl ester \\
\hline 15 & 18.939 & 1.34 & Cyclononasiloxane, octadecamethyl- \\
\hline 16 & 19.582 & 1.59 & 9,12-Octadecadienoic acid, methyl ester \\
\hline 17 & 20.258 & 2.89 & Tetradecanoic acid, Myristic acid \\
\hline 18 & 20.809 & 1.58 & Cyclodecasiloxane, eicosamethyl- \\
\hline 19 & 23.813 & 1.62 & Cyclononasiloxane, octadecamethyl- \\
\hline 20 & 28.282 & 12.55 & Caffein \\
\hline 21 & 29.018 & 1.56 & Cyclononasiloxane, octadecamethyl \\
\hline
\end{tabular}

Table 2(a-c): Showing the identification and relative percentage of volatile compounds in superfine green tea at $90^{\circ} \mathrm{C}$ in 20,40 and 120 minutes time of extraction.

\begin{tabular}{|c|c|c|c|c|c|c|}
\hline Compounds present & \multicolumn{3}{|c|}{ Fine } & \multicolumn{3}{c|}{ Superfine } \\
\hline Extraction time (min) & $\mathbf{2 0}$ & $\mathbf{4 0}$ & $\mathbf{1 2 0}$ & $\mathbf{2 0}$ & $\mathbf{4 0}$ & $\mathbf{1 2 0}$ \\
\hline Myristic acid & 8.74 & 6.85 & 7.87 & 7.92 & 2.09 & 2.89 \\
\hline Palmitic acid & 24.77 & 20.6 & 23.21 & 23.87 & 13.83 & 21.07 \\
\hline Stearic acid & 3.28 & 1.57 & 1.56 & 2.87 & 1.7 & 1.95 \\
\hline Oleic acid & 40.41 & 35.1 & 39.33 & 38.46 & 22.99 & 34.92 \\
\hline Linoleic acid & 1.87 & 0.91 & 0.85 & 1.5 & 0.85 & 2.6 \\
\hline Caffein & 1.82 & 11.4 & 11.47 & 5.79 & 37.7 & 12.55 \\
\hline Phthalic acid & $*$ & $*$ & $*$ & 3.28 & 3.09 & 3.63 \\
\hline 1H-Purin-6-amine & 1.96 & 2.57 & 2.95 & 1.98 & 2.1 & 1.58 \\
\hline
\end{tabular}

Table 3: Relative percentage of some of major compounds which are present in both tea types.

\begin{tabular}{|c|c|c|}
\hline Compound name & Bioactivity & References \\
\hline $\begin{array}{l}\text { Methyltetradecanoate/ } \\
\text { Myristic acid }\end{array}$ & $\begin{array}{l}\text { Antioxidant, Cancer-preventive, } \\
\text { Hypercholesterolemic, Lubricant, } \\
\text { Nematicide }\end{array}$ & [11] \\
\hline $\begin{array}{l}\text { Hexadecanoic acid/ } \\
\text { palmitic acid }\end{array}$ & $\begin{array}{l}\text { Antioxidant, Hypocholesterolemic } \\
\text { Nematicide, Pesticide, Anti-androgenic, } \\
\text { Flavor Hemolytic, 5-Alpha reductase } \\
\text { inhibitor }\end{array}$ & [11] \\
\hline $\begin{array}{l}\text { Octadecanoic acid/ } \\
\text { Stearic acid }\end{array}$ & $\begin{array}{l}\text { 5-Alphareductase inhibitor, Cosmetic, } \\
\text { Flavor, Hypocholesterolemic }\end{array}$ & [11] \\
\hline $\begin{array}{l}\text { 9-Octadecenoic acid/ } \\
\text { oleic acid }\end{array}$ & $\begin{array}{l}\text { Anti-inflammatory, Anti-androgenic, } \\
\text { Cancer preventive, Dermatitigenic, } \\
\text { Hypocholesterolemic, 5-Alpha reductase } \\
\text { inhibitor, Anemiagenic Insectifuge, Flavor }\end{array}$ & [12] \\
\hline \multirow[t]{2}{*}{$\begin{array}{l}\text { 9,12-Octadecadienoic } \\
\text { acid/Linoleic acid }\end{array}$} & $\begin{array}{l}\text { Hypocholesterolemic, Nematicide, } \\
\text { Antiarthritic, Hepatoprotective, Anti- } \\
\text { androgenic, Hypocholesterolemic } \\
\text { Nematicide, 5-Alpha reductase inhibitor, } \\
\text { Antihistaminic, Anticoronary, Insectifuge, } \\
\text { Antieczemic, Antiacne }\end{array}$ & [13] \\
\hline & Anticancerous and diuretic & [14] \\
\hline $\begin{array}{l}\text { 1H-Purine-2,6-dione/ } \\
\text { Caffein }\end{array}$ & $\begin{array}{l}\text { Prevents: liver cirhossis, Type } 2 \text { diabetes, } \\
\text { apnea of prematurity, bronchopulmonary } \\
\text { dysplasia in prenmature infants. } \\
\text { Secondary metabolic products of caffeine: } \\
\text { paraxanthin increase lipolysis, theobromine } \\
\text { is a vasodilator; theophylline acts as a } \\
\text { muscle relaxant, anti-asthmatic. }\end{array}$ & {$[15-18]$} \\
\hline $\begin{array}{l}\text { Phthalic acid, diethyl } \\
\text { ester }\end{array}$ & $\begin{array}{l}\text { Can cause damage to the nervous system } \\
\text { and reproductive system, mutagenic and } \\
\text { carcinogenic. }\end{array}$ & [19] \\
\hline
\end{tabular}

Table 4: Biological activity of some phyto-components identified in the green extracts.
Stearic acid, 9-Octadecenoic acid/oleic acid, 9,12-Octadecenoic acid/Linoleic acid, 1H-PURINE-2,6-DIONE/Caffeine, pthalic acid, diethyl ester and 1H-PURINE-6-AMINE. The percentage release of compounds from these selected tea types varied according to the time of extraction employed, for example the 9-Octadecenoic acid/ oleic acid and Hexadecanoic acid/palmitic acid showed maximum peak area in 20 minutes time of extraction in Fine and Superfine tea types. 1H-PURINE-2,6-DIONE/Caffeine showed maximum peak area in 40 minutes time of extraction in Fine and Superfine varieties. Thus the best time of extraction can be considered in the range of 2040 minutes. Further increase in time leads to decrease in the relative percentage of most of the physiologically important phytochemical compounds. Most of the compounds detected were health benefiting and physiologically important (Table 4) except for pthalic acid/diethyl ester which has been reported to show various toxic effects such as cancer and neurotoxicity etc. However pthalic acid was found in Super fine tea but was not detected in the extracts of Fine tea.

\section{Conclusions}

Present study confirmed presence of various health benefitting compounds using GC-MS. Among various times of extraction employed to tea samples, it was seen that the best time of extraction for the retention of maximum health benefiting compounds such as caffeine, linoleic acid, oleic acid, palmitic acid and etc. was in the range of 20-40 minutes. The present study also suggests presence of some toxic substances in the tea extracts such as pthalic acid which need further in detailed studies.

\section{Acknowledgment}

The authors are highly thankful to Department of Biotechnology, Government of India for financial support.

\section{References}

1. Balentine DA, Harbowy ME, Graham HN (1998) In: Caffeine, Spiller GA (ed.) CRC Press, Boca Raton, USA, 35-68.

2. Chow KB, Kramer I (1990) All the Tea in China, China Books \& Periodicals, San Francisco, USA

3. Ahn WS, Huh SW, Bae SM, Lee IP, Lee JM, et al. (2003) A major constituent of green tea, EGCG, inhibits the growth of a human cervical cancer cell line, CaSki cells, through apoptosis, G(1) arrest, and regulation of gene expression. DNA Cell Biol 22: 217-224

4. Chan PT, Fong WP, Cheung YL, Huang Y, Ho WK, et al. (1999) Jasmine green tea epicatechins are hypolipidemic in hamsters (Mesocricetus auratus) fed a high fat diet. J Nutr 129: 1094-1101.

5. Chen Z, Zhu QY, Tsang D, Huang Y (2001) Degradation of green tea catechins in tea drinks. J Agric Food Chem 49: 477-482.

6. Cheng TO (2006) All teas are not created equal: the Chinese green tea and cardiovascular health. Int J Cardiol 108: 301-308.

7. Kim Y, Goodner KL, Park J, Choi J, Talcott ST (2011) Changes in antioxidant phytochemicals and volatile composition of Camellia sinensis by oxidation during tea fermentation. Food Chem 129: 1331-1342.

8. Jacques RA, Santos JG, Dariva C, Oliveira JV, Caramão EB (2007) GC/MS characterization of mate tea leaves extracts obtained from high-pressure $\mathrm{CO}_{2}$ extraction. J Supercrit Fluid 40: 354-359.

9. Jenkins AJ, Llosa T, Montoya I, Cone EJ (1996) Identification and quantitation of alkaloids in coca tea. Forensic Sci Int 77: 179-189.

10. Bilia AR, Flamini G, Taglioli V, Morelli I, Vincieri FF (2002) GC-MS analysis of essential oil of some commercial Fennel teas. Food Chem 76: 307-310.

11. Kumar RN, Reddy JS, Gopikrishna G, Anand SK (2012) GC-MS Determination of Bioactive Constituents of Cycasbeddomei Cones. International Journal of Pharma and Bio Sciences 3: 344-350.

12. Gopalakrishnan S, Vadivel E (2011) GC-MS Analysis of Some Bioactive 
Citation: Ahmad M, Gani A, Baba WN, Gani A, Wani SM, et al. (2013) Effect of Extraction Time on Physiologically Important Constituents of Green Tea (Camellia sinensis) Using GC/MS. J Food Process Technol 4: 276. doi:10.4172/2157-7110.1000276

Constituents of Mussaenda frondosa Linn. International Journal of Pharma and Biosciences 2: 313-320.

13. Jananie RK, Priya V, Vijayalakshmi K (2011) Determination of Bioactive Components of Cynodon dactylon by GC-MS Analysis. New York Science Journal 4: 16-20.

14. Mathew OP (2011) Apnea of prematurity: pathogenesis and management strategies. J Perinatol 31: 302-310.

15. Kugelman A, Durand M (2011) A comprehensive approach to the prevention of bronchopulmonary dysplasia. Pediatr Pulmonol 46: 1153-1165.
16. Nehlig A, Daval JL, Debry G (1992) Caffeine and the central nervous system mechanisms of action, biochemical, metabolic and psychostimulant effects. Brain Res Brain Res Rev 17: 139-170.

17. van Dam RM (2008) Coffee consumption and risk of type 2 diabetes, cardiovascular diseases, and cancer. Appl Physiol Nutr Metab 33: 1269-1283.

18. Muriel P, Arauz J (2010) Coffee and liver diseases. Fitoterapia 81: 297-305.

19. Kozumbo WJ, Kroll R, Rubin RJ (1982) Assessment of the mutagenicity of phthalate esters. Environ Health Perspect 45: 103-109. 